\title{
Text-contingent color aftereffects: A reexamination
}

\author{
G. KEITH HUMPHREY \\ University of Western Ontario, London, Ontario, Canada \\ DIANE SKOWBO \\ Colby College, Waterville, Maine \\ and \\ LAWRENCE A. SYMONS, ANDREW M. HERBERT, and CHRISTINA L. GRANT \\ University of Western Ontario, London, Ontario, Canada
}

\begin{abstract}
Five experiments reexamined color aftereffects contingent on the semantic properties of text (Allan, Siegel, Collins, \& MacQueen, 1989). The influence of different assessment techniques and the effect of eye movements and overlapping contour information on the induction of color aftereffects by word and nonword letter strings were determined. Experiment 1 showed that no aftereffect was found when a traditional method of assessing color aftereffects was used. Experiments 2 and 4 demonstrated color aftereffects for both words and nonwords, but only when subjects fixated the same locus during induction and testing and only when assessed with the technique described by Allan et al. (1989). If, however, eye movements were made during induction, no color aftereffect was obtained (Experiment 3). Induction to nontext patterns with properties similar to those of text but with fewer overlapping contours resulted in a strong color aftereffect (Experiment 5). These results suggest that the color aftereffect contingent on text is very weak and is not dependent on semantic factors, but that it is a product of induction to local color and orientation information.
\end{abstract}

In 1965, McCollough reported negative color aftereffects contingent on the orientation of induction and test patterns. Now known as the McCollough effect (ME), this visual illusion has been the topic of considerable research, most of which supports McCollough's original contention that the effect occurs at an early stage in the visual system. However, in a recent study, Allan, Siegel, Collins, and MacQueen (1989) obtained results which suggest that higher level, semantic processes might be involved in the effect.

Induction of the $\mathrm{ME}$ involves the alternate viewing of two grating patterns of roughly complementary colors. In McCollough's (1965) original experiments, a grating of orange-and-black vertical stripes alternated with a grating of blue-and-black horizontal stripes. The gratings were viewed at 5 -sec intervals with $1 \mathrm{sec}$ of darkness in between for approximately 2-4 min. When black-andwhite horizontal and vertical gratings were viewed fol-

This research was supported by Colby College and by Grant A1643 from the Natural Sciences and Engineering Council of Canada to G.K.H. This research was presented at the 1993 meeting of the Canadian Society for Brain, Behaviour, and Cognitive Science, Toronto. Thanks are due three anonymous reviewers and the editor Mary Hayhoe for helpful comments on an earlier version of this paper. Correspondence should be addressed to G. K. Humphrey, Department of Psychology, University of Western Ontario, London, ON, Canada N6A 5C2 (e-mail: humphrey@sscl.uwo.ca). lowing a short postadaptation interval, they appeared to be faintly colored in hues approximately complementary to the induction colors. That is, the vertical gratings appeared slightly bluish, and the horizontal gratings, slightly orange. Since then, it has been noted that adaptation using red and green will generally produce a stronger ME (Stromeyer, 1978). Although it takes only minutes to induce the ME, the effect may last minutes, hours, days, and sometimes even weeks or months (see, e.g., Jones \& Holding, 1975; Riggs, White, \& Eimas, 1974).

The characteristics of the ME have led to a general consensus that it is a low-level phenomenon mediated by early visual mechanisms (see reviews by Humphrey, in press; Skowbo, Timney, Gentry, \& Morant, 1975; Stromeyer, 1978). For example, the effect does not transfer from one eye to the other (McCollough, 1965; Murch, 1972); it is specific to retinal orientation, size, and area (Ellis, 1976; Lovegrove \& Over, 1972; McCollough, 1965; Stromeyer, 1972); and attention to the patterns is not necessary for induction (Houck \& Hoffman, 1986) or for detection of the aftereffects (Humphrey, Gurnsey, \& Fekete, 1991). Research done on braindamaged individuals has suggested that the mechanisms responsible for the ME reside in area 17 of the visual cortex or earlier (Goodale, Aglioti, Humphrey, \& Gurnsey, 1992; Humphrey, Goodale, \& Gurnsey, 1991; Savoy \& Gabrielli, 1991). 
Recently, however, Allan et al. (1989) have suggested that the ME might be dependent on higher level, cognitive processes. These authors report color aftereffects contingent on words but not on nonwords composed of the same letters. For instance, subjects viewed the word SIREN colored red alternating with RINSE colored green over a 25-min induction period. This resulted in a color aftereffect. This color aftereffect contingent on text was not found for nonword pairs like ENSRI and RENIS. Given this difference between words and nonwords, Allan et al. claim that the physical characteristics of the words such as line orientation and width are not relevant to their findings. This claim is surprising, because it contradicts previous studies which indicate that the $M E$ is very much dependent on basic physical characteristics of the stimuli.

The text-contingent color aftereffects reported by Allan et al. (1989) appear to be much weaker than aftereffects obtained with gratings and assessed with the same measurement technique (Allan, Siegel, Toppan, \& Lockhead, 1991). Allan et al.'s (1989) technique for measuring the ME resembles the method of constant stimuli (described in detail by Allan et al., 1991). The procedure consists of three phases - a preinduction test, induction, and a postinduction test. The preinduction and postinduction tests are composed of repeated presentations of the induced stimuli, which are colored a desaturated pink or green, and achromatic stimuli. The number of green responses in the preinduction test is compared with the number of green responses postinduction to determine whether an ME has occurred. The resulting function runs across a range of desaturated colors, with white at the midpoint. Following induction, one should observe more green responses to the patterns induced in magenta and fewer green responses to the pattern induced in green when there is an ME (see Experiment 2, Method for a complete description). A visual comparison of the magnitude of the shift in the function from pre- to postinduction tests appears to be much larger for oriented gratings (Allan et al., 1991) than for words (Allan et al., 1989). It is entirely possible that the effect obtained with words would not be found by using more standard techniques for assessing color aftereffects. In our first experiment, we tested the robustness of the text-contingent color aftereffect by attempting to replicate the findings of Allan et al. (1989), using a more conventional method for assessing color aftereffects (described below).

\section{EXPERIMENT 1}

The conditions used by Allan et al. (1989) to produce the text-contingent color aftereffects were unlike those normally used in ME demonstrations. For instance, the luminance of the induction words was relatively dimdimmer, in fact, than that of the test words. Previous work on MEs has shown that the aftereffects show up most vividly on low-luminance achromatic patterns fol- lowing inspection of high-luminance chromatic patterns (White, 1976). Another unusual aspect of the procedure was the fact that only one test word was presented at a time. This restriction precludes the operation of simultaneous contrast - a factor that enhances the likelihood of reporting an ME. Dale, Anderson, and Smith (1976) and Humphrey, Dodwell, and Emerson (1985) had found that significantly more verbal reports of ME hues were given when subjects viewed test patterns in which color contrast was potentially present.

On the other hand, Allan et al. (1989) employed an unusually long adaptation time, 20 or $25 \mathrm{~min}$ instead of the more typical 5-10 min, which might be expected to offset other methodological liabilities. (For descriptions of increases in ME strength with increases in induction time, see Riggs et al., 1974.) Rather than try to estimate the relative contributions of various factors in Allan et al.'s (1989) experiments, however, we simply reasoned that the reported effect should be easy to perceive under optimal stimulus conditions. We therefore attempted to replicate Allan et al.'s (1989) findings by using stimuli typical of traditional ME demonstrations-in particular, high-luminance adaptation patterns and a test figure designed to evoke both aftereffect colors. In addition, we increased the adaptation time slightly, and used a simple, direct assessment method (a single verbal report, with no requirement that subjects guess if colors were not readily apparent in the test pattern). Aside from these factors, we attempted to keep our stimulus conditions as close to theirs as possible.

\section{Method}

Subjects. Twenty-eight people participated in the study. Two of these subjects had seen MEs many times and were aware of the theoretical basis of the experiment. Two others had seen MEs in a classroom demonstration several months earlier, and a $3 \mathrm{rd}$ had been a subject in an ME study conducted 3 years earlier; none of them, however, knew the rationale for the experiment. The remainder were completely naive. Dvorine plates were used to screen all subjects for normal color vision.

Apparatus and Stimuli. Because it can be argued that nonanagram word pairs contain different spatial features (which could be the basis for aftereffects apparently contingent on text), we restricted our experiment to an anagram pair and arbitrarily chose one of the anagram sets used by Allan et al. (1989): ONSET and STONE. We used a laser printer to print these words on white paper in uppercase, bold, Helvetica type. To make the adaptation stimuli, we combined photographic negatives of these prints with Kodak Wratten filters Nos. 58 (green; CIE $x$ and $y$ chromaticity coordinates were approximately 0.303 and 0.638 , respectively) and 32 (magenta; CIE $x$ and $y$ chromaticity coordinates were approximately 0.556 and 0.246 , respectively). In addition, we used Kodak Wratten neutral density filters (No. 96) to attenuate intensity.

Slides made from these materials were shown in a Kodak Carousel Ektagraphic Model B-2 projector; the illuminant was a Type ELH halogen projector lamp. The words were projected onto a piece of frosted glass rear-projection material; when viewed by the subject, they were $0.4^{\circ}$ high and $2^{\circ}$ long (very close to Allan et al.'s, $1989,0.4^{\circ} \times 1.7^{\circ}$ ). We used two adaptation luminance conditions. For 20 subjects (including the 2 experienced ME observers), the character luminance for the green word was 
$240 \mathrm{~cd} / \mathrm{m}^{2}$; that for the magenta word was $219 \mathrm{~cd} / \mathrm{m}^{2}$. Then, for the other 8 subjects plus the two experienced ME observers (who ran a second time), the luminances were reduced to $24 \mathrm{~cd} / \mathrm{m}^{2}$ (green) and $22 \mathrm{~cd} / \mathrm{m}^{2}$ (magenta)-levels comparable to those used by Allan et al. $\left(42\right.$ and $\left.19 \mathrm{~cd} / \mathrm{m}^{2}\right)$.

The test slide was a three-word $x$ five-word matrix in which ONSET and STONE alternated horizontally and vertically. We prepared this slide in a manner like that described for the induction words, except that we used no chromatic filters. The luminance of the characters in the test figure was $54.8 \mathrm{~cd} / \mathrm{m}^{2}$ (the luminance of Allan et al.'s, 1989, achromatic test word was $66 \mathrm{~cd} / \mathrm{m}^{2}$ ).

Procedure. The subjects were run individually in a dark room. At the beginning of the session, they were shown the test stimulus and asked whether they perceived any difference in color between the two words in the matrix. They were told that if any colors did appear, they would be faint and pale. Next, the subjects viewed the two induction words alternating every $10 \mathrm{sec}$ for a total of $30 \mathrm{~min}$ (Allan et al.s, 1989, induction times were 20 or $25 \mathrm{~min}$ ). As in Allan et al. (1989), no instructions were given regarding eye movements. At the end of the induction period, there was a 1-min delay, after which the test slide was shown again. The subjects were then asked the same question as before regarding color perceived in the test word matrix. Finally, subjects were asked whether they had fixated or scanned the adaptation words.

\section{Results}

None of the 28 subjects reported any color in the test words either before or after the adaptation period. It is important to note that these negative reports came not only from naive subjects but also from several individuals with prior ME exposure, all of whom recognized the ME paradigm and had expectations about what they were "supposed" to see. The 2 experienced ME observers scrutinized the test pattern with particular care, but neither saw any color differences in the word matrix. A few subjects commented during postadaptation testing that the word that they fixated looked darker than the others in the matrix. Of the 28 subjects who participated, 12 stated that they had scanned the adaptation words, 10 said that they had fixated, and 6 reported that they had switched intermittently from scanning to fixating.

\section{Discussion}

We were unable to replicate Allan et al.'s (1989) report of a text-contingent ME in this experiment. As noted earlier, our induction and test conditions were different from theirs in a few ways that, according to past research on MEs, should have favored the appearance of such an effect -that is, in luminance of the adaptation stimuli, configuration of the test pattern, and adaptation time.

The present experiment and those of Allan et al. (1989) did differ in one important way-that is, in the assessment procedure. Our subjects were asked to make a single judgment about their perceptual experiences, and a negative report was permitted. Allan et al. (1989) used a forced-choice procedure, in which the subjects had to give a color response on each of 100 trials (or 96 , in the case of one experiment). It might be argued that the latter procedure would be more sensitive to weak effects and, therefore, more likely to reveal evidence for subtle color differences between the test words. We examined this possibility in the second experiment.

\section{EXPERIMENT 2}

We used the measurement technique of Allan et al. (1989) to determine whether this procedure was a particularly sensitive way of detecting weak color aftereffects. There was one notable difference in this experiment, in that we explicitly instructed subjects to fixate the center of the inducing and assessment stimuli. Such instructions were not given in Experiment 1 and had not been given by Allan et al. (1989). There is reason to suspect that matching the locus of fixation on induction and test trials could be an important factor. As noted above, research has demonstrated that the $\mathrm{ME}$ is specific to retinal orientation and location. For example, different orientation-contingent aftereffects can be induced on different parts of the retina (Stromeyer \& Dawson, 1978). Vertical bars may appear pink at one retinal position, but green at another. Stromeyer (1972) demonstrated that such aftereffects disappeared when the test pattern was moved as little as $0.5^{\circ}$ off the induced area and then reappeared when the test pattern was brought within about $0.3^{\circ}$ of the induced area. It was hoped that maintaining fixation would increase the likelihood of obtaining an effect.

\section{Method}

Subjects. The subjects were 3 males and 3 females, ranging in age from 22 to 44 years. They were not screened formally for color deficiencies, but they were not aware of any. All members of the sample were familiar with the ME, although 3 of them were not experienced ME observers at the start of testing.

Apparatus and Stimuli. Stimulus presentation and response recording were controlled by a Macintosh II microcomputer. The stimuli were displayed on a high-resolution Apple 13-in. color monitor. The monitor was located $98 \mathrm{~cm}$ from the subject. The subjects' head movements were restricted by a chinrest equipped with a forehead stop and two lateral head stops.

Luminance and chromaticity (CIE $x$ and $y$ coordinates) were measured with a Minolta Chroma Meter CS-100 and are summarized in Table 1. The luminance values for the green and magenta induction patterns are close to those used by Allan et al. (1989). Each word was presented individually, centered in the middle of the monitor screen against a black background. The text was in uppercase bold Courier type. Each word was $2.7 \times 0.7 \mathrm{~cm}$, subtending $1.6^{\circ} \times 0.4^{\circ}$ of visual angle.

Procedure. Identical preinduction and postinduction tests were administered to determine baseline and test psychometric functions for all subjects. The assessment phases consisted of 100 presentations of two words, SIREN and RINSE; each word was randomly displayed 10 times in each of five colors: light magenta (M2), lightest magenta (M1) (least saturated), achromatic (0), lightest green (G1), and light green (G2). The word remained on the screen until the subject made a binary response - "green" or "pink"-on each presentation. The process was repeated for a second word pair, ONSET and STONE.

The induction phase involved exposing subjects to alternating presentations of the word SIREN in magenta and the word RINSE in green every $3 \mathrm{sec}$ for a period of $25 \mathrm{~min}$. In the second induction session, ONSET was presented in magenta, STONE in green.

The subjects received instruction prior to experimentation and were assessed independently. They were seated in a darkened room and were instructed to adjust the chinrest until it was comfortable. The subjects were asked to fixate the center of the middle letter of each word presented during both the induction and the test phases. 
Table 1

Luminance and Chromaticity Values of the Colors in Experiments 2-5

\begin{tabular}{lcccc} 
& $\begin{array}{c}\text { Luminance } \\
\left(\mathrm{cd} / \mathrm{m}^{2}\right)\end{array}$ & \multicolumn{2}{c}{ Chromaticity } \\
\cline { 4 - 5 } & 0.1 & & $y$ \\
\hline Black & & & \\
Induction Colors & 52.7 & .295 & .590 \\
$\quad$ Green & 18.7 & .367 & .200 \\
$\quad$ Magenta & & & \\
Test Colors & 79.1 & .279 & .305 \\
$\quad$ Light green (G2) & 79.4 & .279 & .302 \\
$\quad$ Lightest green (G1) & 83.7 & .280 & .298 \\
White (0) & 80.0 & .279 & .293 \\
$\quad$ Lightest magenta (P1) & 78.9 & .279 & .291 \\
$\quad$ Light magenta (P2) & & & \\
\hline
\end{tabular}

The RINSE/SIREN word pair was assessed during a single session composed of preinduction assessment, a 25 -min induction period, and a postinduction assessment following $4 \mathrm{~min}$ of normal room illumination. The ONSET/STONE word pair was similarly evaluated at least a week later.

\section{Results}

Figure 1 illustrates the psychometric function relating the mean proportion of "green" responses, $P(\mathrm{G})$, to each of the test colors for the two test phases. Each point in the graph was based on 120 observations: 10 responses from each of 6 subjects over two conditions (RINSE/SIREN and ONSET/STONE).

The baseline functions were similar for both sets of words. The function for the words presented in magenta during induction (SIREN and STONE) showed a slight in-

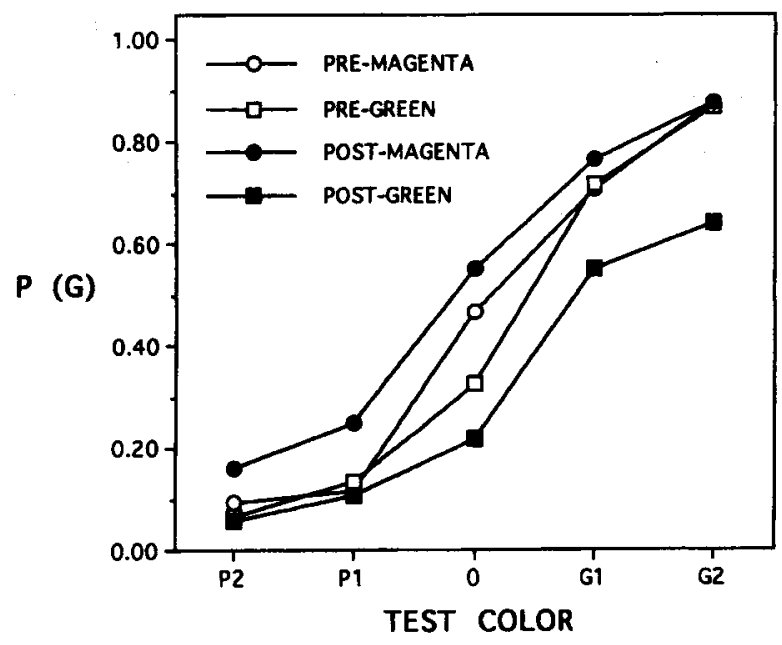

Figure 1. Preinduction and postinduction test psychometric functions obtained in Experiment 2. Because the statistical analyses showed no difference between the two word pairs, the mean of the results for both word pairs is presented. Note that for clarity of presentation these graphs present the range of colors along an evenly spaced scale. The graphic presentation is not meant to imply that the five test stimuli are evenly spaced along an interval scale. Furthermore, because Allan, Siegel, Collin, and MacQueen (1989) used a similar type of presentation, it was felt that having the graphs in similar formats would aid in the comparison of results across studies. crease in green responses during postinduction assessment; the function for words presented in green during induction (RINSE and ONSET) showed a shift downwarda slight decrease in the number of green responses during the posttest.

These shifts in the psychometric functions were evaluated as in Allan et al. (1989). The mean numbers of green responses over the five test colors (which could range from 0 to 10 ) were subjected to a 2 (word pair: RINSE/SIREN or ONSET/STONE) $\times 2$ (induction color: magenta or green) $\times 2$ (assessment phase: preinduction or postinduction) repeated measures analysis of variance (ANOVA). The main effect of assessment phase was not significant $(F<1)$; the main effect of induction color was significant $\left[F(1,5)=7.44, M S_{\mathrm{e}}=414.19, p<.05\right]$, as was the interaction of induction color and assessment phase $\left[F(1,5)=9.97, M S_{\mathrm{e}}=229.69, p<.05\right]$. The mean numbers of green responses to the words in the preinduction assessment did not differ significantly $[F(1,5)$ $<1]$, but it did so in the postinduction assessment $\left[F(1,5)=9.99, M S_{\mathrm{e}}=63.08, p<.05\right]$. The difference indicates that the number of green responses depended on the assessment phase. There was an increase in green responses posttest, relative to baseline, for the magentaadapted words (SIREN and STONE), and a concomitant decrease in posttest green responses for the greenadapted words (RINSE and ONSET).

\section{Discussion}

The results of Experiment 2 indicated that a weak color aftereffect contingent on text can be measured by using Allan et al.'s (1989) procedure. The assessment procedure used in the present experiment appears to be more sensitive than that used in Experiment 1. There is, however, another factor that differed between the experiments. In Experiment 1, following Allan et al., the subjects were not instructed to fixate a particular point on the words during testing and induction. We explicitly instructed subjects to fixate the same point during induction and tests in the present experiment. Given Allan et al.'s conclusion that semantic factors are responsible for the text-contingent color aftereffect, it is perhaps not surprising that eye movements were not controlled. However, if eye movements have an influence on this effect, another explanation can be invoked. The text-contingent MEs reported by Allan et al. could be the result of local adaptation to colored contours presented to different retinal areas, as opposed to "text-contingent" effects. If the effect really is contingent on the semantic properties of the stimuli, eye movements should have no influence. This was examined in the third experiment.

\section{EXPERIMENT 3}

This experiment was designed to explore further the color aftereffect found for words in Experiment 2. The only difference between the two experiments-a change in locus of fixation between induction and test phaseswas implemented in order to assess the possibility that 
the effect was due to adaptation to local color and orientation contingencies.

\section{Method}

Subjects. The subjects were the same as in Experiment 2. This induction procedure was run a minimum of 1 week after Experiment 2. To ensure that the ME from Experiment 2 had dissipated before the subjects participated in this condition, we examined their pretest scores for evidence of the ME, which did not appear to be present (Figure 2). For each of the subsequent experiments, the same procedure was followed: the subjects waited at least a week before induction for each different condition, and their preinduction test scores were evaluated to ensure that no $\mathrm{ME}$ was left from previous induction conditions.

Apparatus and Materials. The stimulus parameters, presentation, and response recording were controlled as in Experiment 2.

Procedure. The procedure was identical to that of Experiment 2 , with the exception of a change in the locus of fixation during the induction and test phases. The subjects were instructed to fixate the center letter of each word during both test phases. During induction, however, fixation alternated from the beginning to the end of each word, as follows. During the first pair of induction trials, the subjects were instructed to fixate the R of RINSE and then the $S$ of SIREN. On the next pair of trials, the direction of gaze was shifted to the E of RINSE and then the N Of SIREN. This pattern was repeated, in this order, for the duration of the induction period ( $25 \mathrm{~min}$ ). Similarly, for the second word pair, subjects focused first on the O of ONSET, and then on the S of STONE, followed by the $\mathrm{T}$ of ONSET and the $\mathrm{E}$ of STONE. The RINSE/SIREN and ONSET/ STONE experiments were run at least 1 week apart. In each case, the induction period was followed by $4 \mathrm{~min}$ of exposure to normal room illumination before the postinduction test.

\section{Results}

The psychometric function relating $P(\mathrm{G})$ to each of the test colors for the two test phases is illustrated in Figure 2. Each point in the graph was based on 120 observations: 10 responses from each of 6 subjects, over two conditions (RINSE/SIREN and ONSET/STONE).

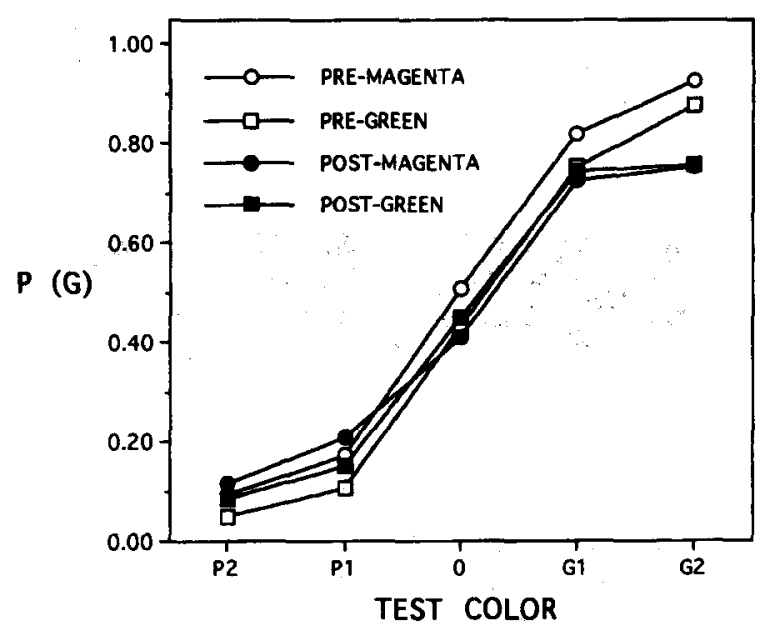

Figure 2. Preinduction and postinduction test psychometric functions obtained in Experiment 3. Because the statistical analyses showed no difference between the two word pairs, the mean of the results for both word pairs is presented.
There was no systematic change in the functions from baseline to test. A 2 (word pair: RINSE/SIREN or ONSET/ STONE) $\times 2$ (induction color: magenta or green) $\times 2$ (assessment phase: preinduction or postinduction) repeated measures ANOVA was conducted on the mean number of green responses over the five test colors, which confirmed no significant effect ( $p>.1$ in all cases).

\section{Discussion}

Eye movements during induction abolished the textcontingent color aftereffect. This argues strongly against the proposal that the text-contingent color aftereffects reported by Allan et al. (1989) were based on semantic factors. Rather, the present results suggest that the color aftereffects found in Experiment 2 were based on local induction by the color and orientation of the elements that make up the individual letters. These findings are consistent with previous research on the ME, which suggests that it is dependent on physical characteristics such as line orientation and width, which activate mechanisms at an early stage in the visual pathway (reviewed by Humphrey, in press; Skowbo et al., 1975; Stromeyer, 1978).

\section{EXPERIMENT 4}

If the aftereffects reported by Allan et al. (1989) were the result of local induction by the color and orientation of the elements of the stimuli, it should be possible to induce an aftereffect for nonwords as well, given appropriate instructions to fixate the stimuli. In the fourth experiment, we assessed the color aftereffects with nonwords when fixation was maintained.

\section{Method}

Subjects. Six subjects participated in the experiment -3 males and 3 females, ranging in age from 22 to 44 years. Five of these subjects had participated in Experiments 2 and 3.

Apparatus and Materials. The apparatus was identical to that of Experiment 2. Two separate nonword pairs were tested: SNOTE (magenta during induction) and OESNT (green during induction) made up one pair, whereas SERIN ${ }^{1}$ (magenta during induction) and RSNIE (green during induction) made up the other.

Procedure. As in Experiment 2, the subjects were asked to fixate the center of the middle letter of each nonword presented during both the induction and the test phases. All other aspects of the procedure were identical to those of Experiment 2.

\section{Results}

Figure 3 illustrates the psychometric function relating $P(\mathrm{G})$ to each of the test colors for the two test phases. Each point was based on 120 observations: 10 responses from each of 6 subjects over two conditions (SNOTE/ OESNT and SERIN/RSNIE). The baseline functions were similar for both sets of nonwords. The function for the nonwords presented in magenta during induction (SNOTE and SERIN) showed an increase in green responses at postinduction assessment; the function for the nonwords presented in green during induction (OESNT and RSNIE) showed a similar shift downward. 


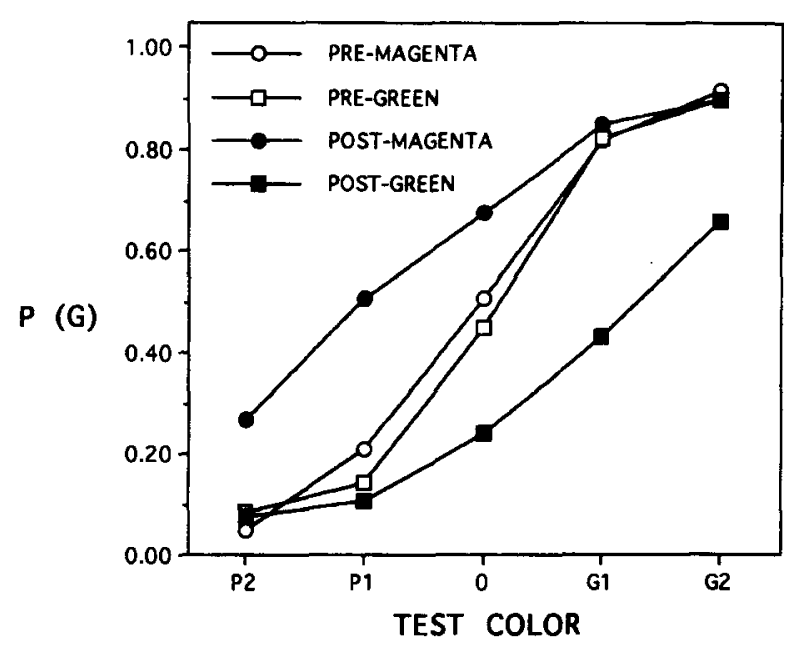

Figure 3. Preinduction and postinduction test psychometric functions obtained in Experiment 4. Because the statistical analyses showed no difference between the two nonword pairs, the mean of the results for both nonword pairs is presented.

The mean numbers of green responses over the five test colors (which could range from 0 to 10 ) were subjected to a 2 (nonword pair: SNOTE/OESNT or SERIN/RSNIE) $\times 2$ (induction color: magenta or green) $\times 2$ (assessment phase: preinduction or postinduction) repeated measures ANOVA. The main effect of assessment phase was not significant; the main effect of induction color was significant $\left[F(1,5)=20.4, M S_{\mathrm{e}}=954, p<.01\right]$, as was the interaction of induction color and assessment phase $\left[F(1,5)=22.1, M S_{\mathrm{e}}=752, p<.01\right]$. The mean numbers of green responses to the nonwords in the preinduction assessment did not differ significantly $[F(1,5)$ $<1]$, but did so in the postinduction assessment $[F(1,5)=$ $\left.24.7, M S_{\mathrm{e}}=69, p<.01\right]$. The difference indicates that the number of green responses depended on the assessment phase. There was an increase in $P(\mathrm{G})$ posttest, relative to baseline, for the magenta-adapted nonwords (SNOTE and SERIN), and there was a decrease in $P(G)$ posttest for the green-adapted nonwords (OESNT and RSNIE).

\section{Discussion}

The results support our contention that the textcontingent color aftereffects reported by Allan et al. (1989) are the result of induction by local orientation and color contingencies and are not dependent on the semantic properties of the stimuli. The color aftereffects induced with nonwords are at least of the magnitude of those found with words in Experiment 2.

\section{EXPERIMENT 5}

If the color aftereffect reported by Allan et al. (1989) is strictly the result of induction by local contour information, we should be able to produce MEs with nontext patterns having physical characteristics similar to those of the words and nonwords used in Experiments 2, 3, and 4. In Experiment 5, we examined this possibility by using two chevron-like patterns (see Figure 4) as inducing stimuli with physical characteristics that were in many ways similar to those of the words and nonwords. In the case of the text-based stimuli, some of the local orientations would be the same in both inducing patterns. The letters making up the words are composed of horizontally, vertically, and diagonally oriented straight components, and of a few curved segments. The middle letter of RINSE, for example, which was induced in green, was composed of two vertical lines and one diagonal. The middle letter of SIREN, which was induced in magenta, was composed of one vertical, one diagonal, and two horizontal lines, along with a curved segment. It is possible that two inducing stimuli with shared orientation characteristics in different colors may have contributed to the weakness of the effect. The chevron-like patterns of Figure 4 were designed so that, with central fixation, the contours in one pattern were orthogonal to those in the other pattern. According to our argument, this should produce a stronger ME than the MEs found in Experiments 2 and 4, in which there should have been some local cancellation of the aftereffects because of similarly oriented contours being presented to the same retinal region in two colors.

\section{Method}

Subjects. The 6 subjects from Experiment 4 participated in Experiment 5.

Apparatus and Materials. The apparatus was identical to that in Experiments 2, 3, and 4. The chevron-like patterns (see Figure 4) were composed of diagonal line segments of the same width as the line segments making up the letters of the stimuli in Experiments 2,3, and 4. The overall size of the patterns and the visual angle they subtended were identical to those of the words used in Experiments 2, 3, and 4. The chevron-like pattern pointing down was presented in magenta during induction; that pointing up was presented in green.

Procedure. As in Experiments 2 and 4, the subjects were asked to fixate the center of the patterns during the induction and test trials. All other aspects of the procedure were identical to those of Experiment 2.
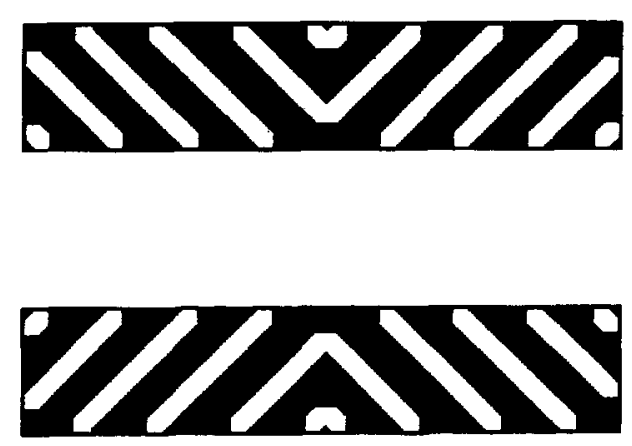

Figure 4. The chevron-like stimuli used in Experiment 5. The chevron-like pattern pointing down was presented in magenta during induction; that pointing up was presented in green. 


\section{Results}

Figure 5 illustrates the psychometric function relating $P(G)$ to each of the test colors for the two test phases. Each point in the graphs is based on 60 observations: 10 responses from each of 6 subjects per pattern for each color. The baseline functions were similar for both patterns. The function for the pattern presented in magenta during induction showed a large increase in green responses at postinduction assessment; the function for the pattern presented in green during induction shows a large downward shift in $P(\mathrm{G})$.

The mean numbers of green responses over the five test colors (which could range from 0 to 10 ) were subjected to a 2 (induction color: magenta or green) $\times 2$ (assessment phase: preinduction or postinduction) repeated measures ANOVA. The main effect of assessment phase was not significant; the main effect of induction color was significant $\left[F(1,5)=352, M S_{\mathrm{e}}=7.5, p<.01\right]$, as was the interaction of induction color and assessment phase $\left[F(1,5)=444, M S_{\mathrm{e}}=7.6, p<.01\right]$. The number of green responses depended on the assessment phase in such a way that there was an increase in $P(\mathrm{G})$ posttest for the magenta-induced pattern, relative to baseline, and a decrease in $P(G)$ posttest for the green-induced pattern.

\section{Discussion}

The results show that small, nontext patterns can be used to induce an ME. Although sharing many of the physical characteristics of the text-based stimuli, the chevron-like patterns induced a much stronger aftereffect than did the text-based patterns (compare Figure 5 with Figures 1 and 3). The composition of the patterns ensured that, with central fixation, orthogonally oriented contours appeared in different colors in different parts of the retina during induction. The results support our con-

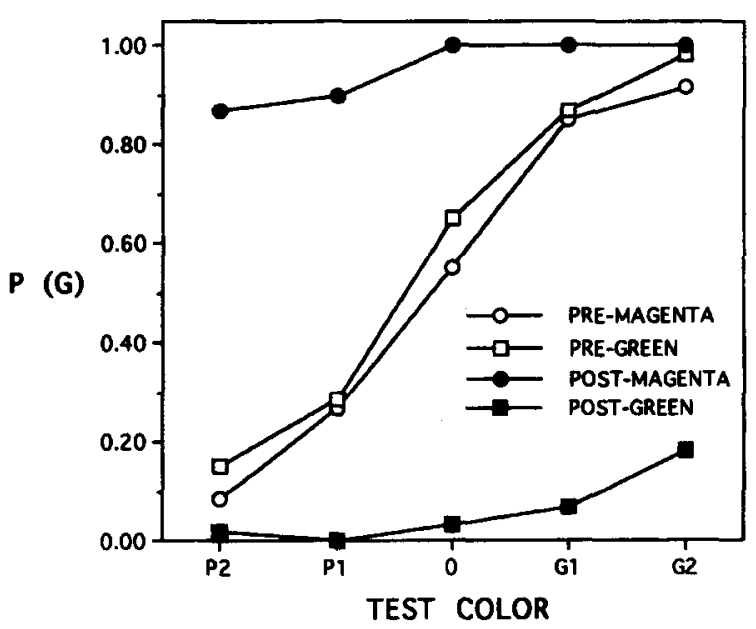

Figure 5. Preinduction and postinduction test psychometric functions obtained in Experiment 5 after induction with the chevron-like patterns. jecture that the weak MEs induced with text-based stimuli occurred because of the overlap in the orientations of the line segments making up the letters in an inducing word or nonword pair.

We should point out that, while the patterns we used are not strictly chevrons, there has been some debate in the literature over the mechanisms that are adapted with similar patterns. White and Riggs (1974; see also Riggs, 1973) have proposed that such patterns contain angles and adapt mechanisms that are sensitive to the direction of departure from a straight line. Many other results suggest, however, that induction with angled or curved line patterns can be accounted for in terms of adaptation of mechanisms sensitive to line orientation and retinal position (e.g., Crassini \& Over, 1975; Hayman \& Allan, 1980; Sigel \& Nachmias, 1975). White and Riggs argued that the color aftereffects cannot be due to local color and orientation contingencies, because they obtained color aftereffects when subjects slowly scanned the patterns. Others, however, have not been able to obtain effects under similar induction conditions in which the pattern moved relative to the retina (Mackay \& MacKay, 1974; Sigel \& Nachmias, 1975; Stromeyer, 1974). Furthermore, Hayman and Allan (1980) found that there were changes in the hue reported as a result of shifts in the locus of fixation on test figures and that these changes were those that were expected on the basis of spatially localized aftereffects. On balance, it appears that chevron-like patterns produce highly localized orientation-contingent aftereffects. We also feel that the size and structure of the patterns we used were sufficiently different from those used previously that this debate is tangential to the present concerns.

Finally, we should note that the chevron-like patterns are only one way of testing for the effects of local orthogonality. Although the patterns we used were similar to the words used in Experiments 2 through 4 in terms of the size of the overall pattern and the width of the elements, other characteristics made them different. The most notable difference occurred in the repetitive structure of the patterns, which was not present in the words and nonwords. Another way of testing for the effects of local orthogonality, as suggested by an anonymous reviewer, would be to contrast the effects obtained with anagrams that had similar feature orientations in the same retinal locus with those obtained with anagrams that had orthogonal feature orientations in the same retinal locus.

\section{GENERAL DISCUSSION}

The results of these five experiments provide an alternative explanation for the text-contingent color aftereffects reported by Allan et al. (1989). The negative results of Experiment 1 suggest that the text-based color aftereffects can only be observed under certain induction and testing conditions; they are not strong effects and do not appear under traditional ME induction and testing 
conditions. A weak ME can be found if assessed with the technique of Allan et al. (1989), but subjects had to maintain fixation during induction and testing (Experiment 2). However, the results of Experiment 3, which show that the aftereffect is not found when subjects move their eyes during induction, suggest that the effect is not dependent on the semantic properties of the stimuli. The color aftereffect demonstrated with fixation on nonword pairs in Experiment 4 directly refutes the findings and semantic contingency hypothesis of Allan et al. (1989).

It appears instead that the effect reported by Allan et al. (1989) was entirely contingent on the local color and orientation of line segments making up the individual induction letters. The results of Experiment 5 bolster this argument, because the chevron-like patterns, which had orthogonally oriented contours under central fixation, produced very strong color aftereffects. Collectively, these findings are consistent with previous research on the ME, which suggests that it is dependent on physical characteristics, such as line orientation and line width, that activate mechanisms at an early stage of visual processing (Humphrey, in press; Skowbo et al., 1975; Stromeyer, 1978). These results are also consistent with those of another study performed recently in our lab, in which we found that the color aftereffects contingent on form reported by Siegel, Allan, and Eissenberg (1992) were also the result of induction by local contour and orientation contingencies (Humphrey, Herbert, Symons, \& Kara, 1994; see also Broerse \& Grimbeek, 1994).

Whereas local effects may be the explanation for the ME observed in the present experiments, it cannot explain why Allan et al. (1989) did not produce identical findings when dealing with nonword stimuli. As we have suggested above, the discrepancy in findings may be explained by the lack of fixation instructions in the experiments of Allan et al. We can only speculate that subjects may be more likely to increase eye movements in response to nonwords as opposed to words when points of fixation are not specified.

Another factor that differentiated Experiments 2-5 from Allan et al. (1989) was that we tested a small number of experienced observers for subjects, whereas Allan et al. tested larger numbers of experimentally naive observers. We believe that our use of experienced observers was justified, because the experiments were quite demanding in terms of holding fixation steady for an extended period of time. There is some evidence that experienced observers may be more sensitive to weak aftereffects. Dodwell and O'Shea (1987, note added in proof) found that experienced observers detected weak aftereffect colors following induction with nonstructured patterns that were not detected by naive observers. Given the weakness of the effects with the text-based stimuli, we believed that the use of experienced observers would increase the likelihood of detecting the aftereffects. However, the use of experienced observers may also raise the possibility of bias. Although it is expected that each observer conducted the experiments impartially, in fact each entered the study with the expectation that a color aftereffect would not be found with "words," given the results of Experiment 1. That the subjects were aware of the hypothesis of this study, as is common in psychophysical experiments, may actually lend credence to these unexpected results.

It appears from the present research that the mechanisms that underlie the ME operate on a local, retinotopic basis and are not influenced by semantic factors. Humphrey (in press) has recently reviewed much of the ME research and has concluded, as have many others, that most findings indicate that the ME is a low-level phenomenon mediated by low-level visual mechanisms. We can ask the question, however, whether the ME plays any functional role in vision. One suggestion, made by Dodwell and Humphrey (1990, 1993; Humphrey, in press), is that the ME is a reflection of the operation of a mechanism that maintains the internal representation of the long-term statistical relationship between color and various spatial parameters. They proposed that the long-term correlation between color and contour orientation, for example, is zero. To maintain this condition in the presence of the artificially high correlation induced between color and contour orientation during $\mathrm{ME}$ generation, the system recalibrates and, in effect, decorrelates color and orientation (see also Barlow, 1990). On this account, the ME reflects the operation of processes that adjust the internal representation of the world to conform to the long-run statistical properties of the external environment.

\section{REFERENCES}

Allan, L. G., Siegel, S., Collins, J. C., \& MacQueen, G. M. (1989). Color aftereffect contingent on text. Perception \& Psychophysics, 46, 105-113.

Allan, L. G., Siegel, S., Toppan, P., \& Lockhead, G. R. (1991). Assessment of the McCollough effect by a shift in psychometric function. Bulletin of the Psychonomic Society, 29, 21-24.

BarLow, H. B. (1990). A theory about the functional role and synaptic mechanism of visual after-effects. In C. Blakemore (Ed.), Vision: Coding and efficiency (pp. 363-375). Cambridge: Cambridge University Press.

Broerse, J., \& GrimbeeK, P. (1994). Eye movements and the associative basis of contingent colored aftereffects: A comment on Siegel, Allan, and Eissenberg. Journal of Experimental Psychology: General, 123, 81-85.

CrassinI, B., \& OVER, R. (1975). Curvature-specific color aftereffects. Perception \& Psychophysics, 17, 398-404.

Dale, A., Anderson, D., \& SMith, M. (1976). McCollough effect: Specificity of induction and line width. Perceptual \& Motor Skills, 42, 1211-1218.

Dodwell, P. C., \& HumphreY, G. K. (1990). A functional theory of the McCollough effect. Psychological Review, 97, 78-89.

Dodwell, P. C., \& HumphreY, G. K. (1993). What is important about McCollough effects? A reply to Allan and Siegel. Psychological Review, 100, 347-350.

DODWELl, P. C., \& O'SHEA, R. P. (1987). Global factors generate the McCollough effect. Vision Research, 27, 569-580.

ELLIS, S. R. (1976). Orientation constancy of the McCollough effect. Perception \& Psychophysics, 19, 183-192. 
Goodale, M. A., Aglioti, S., Humphrey, G. K., \& Gurnsey, R. (1992, November). The McCollough effect reveals orientation discrimination in a case of cortical blindness. Paper presented at the annual meeting of the Psychonomic Society, St. Louis.

Hayman, C. A. G., \& AllaN, L. G. (1980). A reevaluation of anglecontingent aftereffects. Perception \& Psychophysics, 28, 61-67.

Houck, M. R., \& HofFman, J. E. (1986). Conjunction of color and form without attention: Evidence from an orientation-contingent color aftereffect. Journal of Experimental Psychology: Human Perception \& Performance, 12, 186-199.

HuMPHREY, G. K. (in press). The McCollough effect: Misperception and reality. In V. Walsh \& J. Kulikowsi (Eds.), Perceptual constancies: Why things look as they do. Cambridge: Cambridge University Press.

Humphrey, G. K., Dodwell, P. C., \& Emerson, V.F. (1985). The roles of pattern orthogonality and color contrast in the generation of pattern-contingent color aftereffects. Perception \& Psychophysics, 38, 343-353.

Humphrey, G. K., Goodale, M. A., \& GuRnsey, R. (1991). Orientation discrimination in a visual form agnosic: Evidence from the McCollough effect. Psychological Science, 2, 331-335.

HuMPhrey, G. K., GurnSEY, R., \& FEKETE, E. (1991). Rapid discrimination of McCollough effects. Perception, 20, 467-482.

HuMPHREY, G. K., HERBERT, A. M., SyMONS, L. A., \& KARA, S. (1994). McCollough effect to "form": A local phenomenon. Journal of Experimental Psychology: General, 123, 86-90.

JoNES, P. D., \& HoldiNG, D. H. (1975). Extremely long-term persistence of the McCollough effect. Journal of Experimental Psychology: Human Perception \& Performance, 1, 323-327.

LOVEGROVE, W. J., \& OVER, R. (1972). Color adaptation of spatial frequency detectors in the human visual system. Science, 176, 541543.

MACKAY, D. M., \& MACKAY, V. (1974). Do curvature-contingent chromatic aftereffects require "detectors" for curvature? Vision Research, 14, 1285-1288.

McCollough, C. (1965). Color adaptation of edge-detectors in the human visual system. Science, 149, 1115-1116.

MURCH, G. M. (1972). Binocular relationships in a size and color orientation specific aftereffect. Journal of Experimental Psychology, 93, 30-34.

RIGGs, L. A. (1973). Curvature as a feature of pattern vision. Science, 181, 1070-1072.
Riggs, L. A., White, K. D., \& Eimas, P. D. (1974). Establishment and decay of orientation-contingent aftereffects of color. Perception \& Psychophysics, 16, 535-542.

SavoY, R. L., \& GabrielLi, J. D. E. (1991). Normal McCollough effect in Alzheimer's disease and global amnesia. Perception \& Psychophysics, 49, 448-455.

Siegel, S., Allan, L. G., \& EissenberG, T. (1992). The associative basis of contingent color aftereffects. Journal of Experimental Psychology: General, 121, 79-94.

Sigel, C., \& Nachmias, J. (1975). A re-evaluation of curvaturespecific chromatic aftereffects. Vision Research, 15, 829-836.

Skowbo, D., Timney, B. N., Gentry, T. A., \& Morant, R. B. (1975). McCollough effects: Experimental findings and theoretical accounts. Psychological Bulletin, 82, 497-510.

STROMEYER, C. F., III (1972). Contour contingent color aftereffects: Retinal area specificity. American Journal of Psychology, 85, 227235.

Stromeyer, C. F., III (1974). Curvature detectors in human vision? Science, 184, 1199-1200.

STROMEYER, C. F. [III] (1978). Form-color aftereffects in human vision. In R. Held, H. W. Leibowitz, \& H. L. Teuber (Eds.), Perception: Handbook of sensory physiology (Vol. 8, pp. 97-142). New York: Springer-Verlag.

StromeyER, C. F. III, \& DAwson, B. M. (1978). Form-colour aftereffects: Selectivity to local luminance contrast. Perception, 7, 407415.

WHITE, K. D. (1976). Luminance as a parameter in establishment and testing of the McCollough effect. Vision Research, 16, 297-302.

WhITE, K. D., \& RigGs, L. A. (1974). Angle-contingent color aftereffects. Vision Research, 14, 1147-1154.

\section{NOTE}

1. Subsequent to conducting our experiments, we discovered that serin means a small European finch related to the canary, according to Webster's ( 7 th ed.). None of the subjects was aware, during testing, that serin is a word. Furthermore, the fact that there was no difference in the two nonword pairs suggests that this was not a factor.

(Manuscript received July 7, 1993; revision accepted for publication March 29, 1994.) 